\title{
Genetic characterisation of the emerging invasive Neisseria meningitidis serogroup Y in Sweden, 2000 to 2010
}

S Thulin Hedberg' ${ }^{1}$, B Törös ${ }^{1}$, H Fredlund ${ }^{1}$, P Olcén ${ }^{1}$, P Mölling (paula.molling@orebroll.se) ${ }^{1}$

1. National Reference Laboratory for Pathogenic Neisseria, Department of Laboratory Medicine, Clinical Microbiology, Örebro University Hospital, Örebro, Sweden

Citation style for this article:

Thulin Hedberg S, Törös B, Fredlund H, Olcén P, Mölling P. Genetic characterisation of the emerging invasive Neisseria meningitidis serogroup Y in Sweden, 2000 to 2010. Euro Surveill. 2011;16(23):pii=19885. Available online: http://www.eurosurveillance.org/ViewArticle.aspx?Articleld=19885

Article published on 9 June 2011

Neisseria meningitidis serogroups $\mathrm{B}$ and $\mathrm{C}$ have been responsible for the majority of invasive meningococcal disease in Europe. Recently, an increase of N. meningitidis disease due to serogroup $Y$ has been noted in Sweden (in 2010, the proportion was 39\%, with an incidence of 0.23 per 100,000 population), as well as in other northern European countries. We aimed to investigate the clonal pattern of the emerging serogroup $Y$ in Sweden during 2000 to 2010. The serogroup $Y$ isolates identified during this time $(n=85)$ were characterised by multilocus sequence typing and sequencing of the fet $A$, fHbp, penA, porA and porB genes. The most frequent clone (comprising 28 isolates) with identical allele combinations of the investigated genes, was partly responsible for the observed increased number of $N$. meningitidis serogroup $Y$ isolates. It was sulfadiazine resistant, with genosubtype $P_{1} .5-2,10-1,36-2$, sequence type 23 , clonal complex 23 , porB allele 3-36, fet $A$ allele $\mathrm{F}_{4-1}$, $f H b p$ allele 25 and penA allele 22. The first case with disease due to this clone was identified in 2002: there was a further case in 2004, six during 2006 to 2007 , eight during 2008 to 2009 , with a peak of 12 cases in 2010. An unusual increase of invasive disease in young adults (aged 20-29 years) caused by this clone was shown, but no increase in mortality rate was observed.

\section{Introduction}

Neisseria meningitidis is a Gram-negative bacterium that is an obligate commensal of humans: it is carried without symptoms in the pharynx by about $10 \%$ of the general population. It can occasionally cause severe infection, mainly septicaemia and meningitis, when it crosses the epithelial barrier to invade the bloodstream and the meninges [1]. Meningococcal disease is most common in infants but the carriage rate is highest in young adults. Despite treatment and modern intensive care, the disease is still fatal in about $10 \%$ of cases [2].

N. meningitidis is divided into different serogroups, depending on the biochemical composition of the capsule surrounding the bacterium, but only isolates belonging to the $A, B, C, W-135, X$ and $Y$ serogroups have a major role in causing disease [1]. Different hyperinvasive lineages of $N$. meningitidis cause disease with a unique epidemiology and the distribution of the serogroups is highly regional [2]. In Africa, especially in the so-called meningitis belt, the disease is mostly caused by serogroup $A$, but serogroups $C$, $W$-135 and $X$ may also be involved. In Asia, serogroup $A$ is also the most common serogroup. In Europe, North and South America and Australia, serogroups B and $C$ have for decades been the dominating serogroups [3]. In the mid-1990s, however, the incidence of disease due to serogroup $Y$ increased substantially in the United States (US) and today one third of the N. meningitidis infections in that country are caused by this serogroup $[4,5]$. During the last decade, there has also been an increase of meningococcal disease caused by serogroup $Y$ in Canada and Colombia $[6,7]$. In addition, some northern European countries, displayed higher proportions of disease caused by this serogroup: for example, in Norway in 2009 and 2010, the proportion was $31 \%$; in Finland, it was $38 \%$ in 2010 [8].

\section{FIGURE 1}

Incidence of invasive Neisseria meningitidis caused by serogroups B, C, Y and W-135, Sweden, 2000-2010

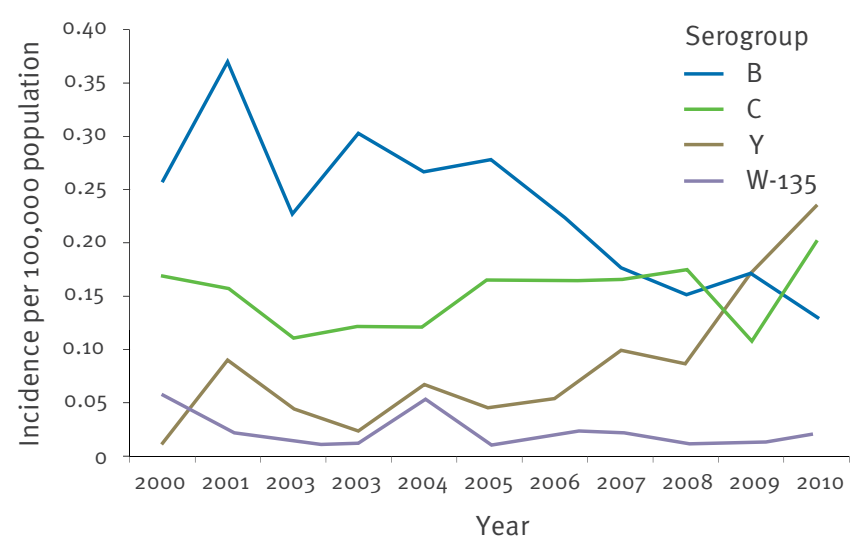

${ }^{a}$ In 2010, the number of $N$. meningitidis isolates were: 12 (serogroup B), 19 (serogroup C), 22 (serogroup Y) and 2 (serogroup $\mathrm{W}-135$ ). 
Until recently, meningococcal disease in Sweden has followed the European serogroup distribution pattern, with the disease being caused mainly by serogroups $\mathrm{B}$ and $\mathrm{C}$. However, the incidence of invasive N. meningitidis caused by serogroup $Y$ started to increase in Sweden in the mid-2000s, rising from 0.04 per 100,000 population in 2005 to 0.23 per 100,000 population in 2010 (Figure 1). The reasons for the dramatic shift in serogroup distribution are unknown. One explanation could be that a new serogroup $Y$ clone has been introduced in Sweden. To investigate this, extensive genetic characterisation must be conducted.

The European Meningococcal Disease Society has published a designation scheme for genetic characterisation of $N$. meningitidis based on serogroup, genosubtype based on analysis of the variable regions of the porA gene (encoding porin $A$, an outer membrane protein), fet $A$ (encoding FetA, an iron-regulated outer membrane protein), sequence type (ST) and clonal complex (cc), the last two being determined by multilocus sequence typing (MLST) [9]. In addition, other gene targets such as porB (encoding porin $B$, another outer membrane protein), penA (encoding penicillinbinding protein 2 , involved in penicillin susceptibility) and $\mathrm{fHbp}$ (encoding factor $\mathrm{H}$-binding protein, which is a promising novel vaccine antigen) can be used for more discriminatory characterisation.

The aim of this study was to investigate by genetic characterisation the clonal pattern of the emerging invasive $N$. meningitidis serogroup $Y$ isolates in Sweden between 2000 and 2010.

\section{Methods}

\section{Bacterial isolates}

The Swedish Institute for Infectious Disease Control (SMI) is mandatorily notified of all invasive cases of meningococcal disease by clinicians, using the European Union

\section{TABLE 1}

Primers for all target genes used in real-time PCR to characterise invasive Neisseria meningitidis serogroup Y isolates, Sweden, 2000-2010 (n=85)

\begin{tabular}{|c|c|c|c|c|}
\hline Target gene & Primer & Primer sequence $\left(5^{\prime} \rightarrow 3^{\prime}\right)$ & $\begin{array}{l}\text { Length of amplicon a } \\
\text { (base pairs) }\end{array}$ & Reference \\
\hline \multirow{2}{*}{$a b c Z$} & $\mathrm{P}_{2} \mathrm{C}$ & TCCCCGTCGTAAAAAACAATC & \multirow{2}{*}{856} & \multirow{14}{*}[14]{} \\
\hline & $\mathrm{P}_{1} \mathrm{C}$ & TGTTCCGCTTCGACTGCCAAC & & \\
\hline \multirow{2}{*}{$a d k$} & $\mathrm{P}_{1} \mathrm{~B}$ & CCAAGCCGTGTAGAATCGTAAACC & \multirow{2}{*}{697} & \\
\hline & $\mathrm{S} 2$ & CAATACTTCGGCTTTCACGG & & \\
\hline \multirow{2}{*}{ aroE } & $\mathrm{P}_{1} \mathrm{~B}$ & TTTGAAACAGGCGGTTGCGG & \multirow{2}{*}{835} & \\
\hline & $\mathrm{P}_{2} \mathrm{~B}$ & CAGCGGTAATCCAGTGCGAC & & \\
\hline \multirow{2}{*}{ fumc } & S1 & TCCGGCTTGCCGTTTGTCAG & \multirow{2}{*}{530} & \\
\hline & $\mathrm{S}_{2}$ & TTGTAGGCGGTTTTGGCGAC & & \\
\hline \multirow{2}{*}{$g d h$} & $\mathrm{P}_{1} \mathrm{~B}$ & CTGCCCCCGGGGTTTTCATCT & \multirow{2}{*}{677} & \\
\hline & $\mathrm{P}_{2} \mathrm{~B}$ & TGTTGCGCGTTATTTCAAAGAAGG & & \\
\hline \multirow{2}{*}{$p d h C$} & $\mathrm{P}_{1} \mathrm{~B}$ & CCGGCCGTACGACGCTGAAC & \multirow{2}{*}{818} & \\
\hline & $\mathrm{P}_{2} \mathrm{~B}$ & GATGTCGGAATGGGGCAAACA & & \\
\hline \multirow{2}{*}{$p g m$} & $\mathrm{P}_{1}$ & CTTCAAAGCCTACGACATCCG & \multirow{2}{*}{1,186} & \\
\hline & $\mathrm{S} 2 \mathrm{~A}$ & GGTGATGATTTCGGTYGCRCC & & \\
\hline \multirow{3}{*}{ penA } & $1 \mathrm{~F}$ & ATCGAACAGGCGACGATGTCb & \multirow{2}{*}{697} & {$[15]$} \\
\hline & ModGcDown3-R & CGGGGATATAACTGCGGCCGTC ${ }^{\circ}$ & & [16] \\
\hline & $1 R^{d}$ & GATTAAGACGGTGTTTTGACGGc & 512 & [15] \\
\hline \multirow{2}{*}{ fet $A$} & $\mathrm{~S} 12$ & TTCAACTTCGACAGCCGCCTT' & \multirow{2}{*}{429} & \multirow{2}{*}[17]{} \\
\hline & S15 & TTGCAGCGCGTCRTACAGGCG' & & \\
\hline \multirow{3}{*}{$f H b p$} & $\mathrm{~F}$ & TGACCTGCCTCATTGATGC & \multirow{2}{*}{950} & \multirow{2}{*}{ [18] } \\
\hline & $\mathrm{R}$ & CGGTAAATTATCGTGTTCGGACGGC & & \\
\hline & ${ }_{5} \mathrm{CE} 2086 \mathrm{mod}^{\mathrm{d}}$ & TATGACTAGGAGYAAACCTG & 882 & Modified from [19] \\
\hline \multirow{2}{*}{ por $B$} & S1 & GCAGCCCTTCCTGTTGCAGC & \multirow{2}{*}{973} & \multirow{2}{*}[17]{} \\
\hline & $\mathrm{S}_{2}$ & TTGCAGATTAGAATTTGTG & & \\
\hline
\end{tabular}

a Amplicon lengths in MC58 [13].

b Universal forward sequence adaptor (5'-GTTTTCCCAGTCACGACGTTGTA-3') added to the 5'-end of the primer [15].

' Universal reverse sequence adaptor (5'-TTGTGAGCGGATAACAATTTC-3') added to the 5'-end of the primer [15].

d The penA1R and fHbp 5CE2086mod primers were used to complement the penA ModGcDown3-R and fHbp F primers, respectively, due to polymorphism in the annealing sites. 
case definition [10] and the corresponding isolates are sent to the Swedish National Reference Laboratory for Pathogenic Neisseria. Basic epidemiological data (age, sex, area of residence and clinical site of isolation) are gathered routinely for all isolates from cases. We analysed all invasive $N$. meningitidis serogroup $Y$ isolates in Sweden between 2000 and $2010(n=85)$ from a total of 637 invasive isolates collected during this time period. The isolates were from clinical specimens of cerebrospinal fluid $(n=11)$, blood $(n=73)$ and joint fluid $(n=1)$ and were cultured on chocolate agar.

\section{Serogrouping and genosubtyping}

The isolates were routinely serogrouped by co-agglutination [11] and subsequently genosubtyped (analysis of porA variable regions), as previously described [12]. Antibiotic susceptibility was determined using Etest (AB Biodisk, Sweden). As a reference, a serogroup B strain $M C_{5} 8$ [13] was included.

\section{Real-time PCR}

The DNA used for amplification and sequencing was prepared from bacterial colonies by boiling or using the Bullet BUGS'n BEADS kit (NorDiag ASA, Norway). The genes targeted in MLST ( $a b c Z$, adk, aroE, fumC, $g d h, p d h($ and $p g m$ ), together with fetA, $f H b p$, penA and porB, were amplified by real-time PCR using the PCR primers shown in Table 1. In each PCR run, $M_{5} 8$ was used as positive control.

\section{DNA sequencing and sequence alignment}

The PCR products were purified by vacuum filtration and subsequently cycle sequenced. The primers used for sequencing of the genes used for MLST were those recommended [14], except for $p d h C$, for which the PCR primer $\mathrm{P}_{2} \mathrm{~B}$ used to amplify $p d h C$ was used for sequencing. The $f H b p$ gene was sequenced with the respective PCR primers as well as gna1870 S2 and gna1870 S3 [18]. The fet $A$ and penA genes were sequenced with the oligonucleotide sequences of the adaptors (Table 1 ) attached to the respective PCR primer. When penA1R was used, it was used for both PCR and sequencing. The por $B$ gene was sequenced with the $P C R$ primers as well as $8 \mathrm{U}, 8 \mathrm{~L}$ [17] and PB7f2 (5'-TYGGCAACGTAACGC-3'), where $\mathrm{Y}$ is $\mathrm{C}$ or T.

The sequence alignments were assembled using ChromasPro software version 1.33 (Technelysium Pty Ltd, Australia). The different sequences were assigned allele numbers using the $N$. meningitidis sequence query database [20]. A clone was defined by all isolates sharing the same genosubtype, ST, sulfadiazine susceptibility and combination of penA, fetA, $f H b p$ and porB alleles.

\section{Data analysis}

We evaluated whether each sequenced gene could be used to discriminate between strains, on the basis of Simpson's index of diversity [21]. The discrimination (D)-index determines the probability that two randomly picked strains will be separated into different typing groups. A high D-index (close to 1) divides the isolates into many small groups (high discriminatory capacity), whereas a low D-index (close to o) indicates that the typing target only divides the isolates into a few large groups (low discriminatory capacity). Confidence intervals of $95 \%$ were calculated [22].

Minimum-spanning trees of the MLST profile data were created to investigate the ST clusters. The links were determined with Prim's algorithm and the clustering was created with the BURST (based on related STs) algorithm [23]. The porA genosubtypes and porB, penA, fet $A$ and $f H b p$ alleles were subsequently distributed over the different STs in the minimum-spanning trees, to describe the variation of different genes within each ST.

\section{Results}

\section{Genetic characterisation}

Using genosubtyping, MLST, and porB, fetA, penA and $f H b p$ analysis, we found three distinctive clones comprising more than five isolates each. The most frequent clone $(n=28)$, referred to as Clone YI, was sulfadiazine

\section{FIGURE 2}

Distribution over time of the three most common invasive Neisseria meningitidis serogroup Y clones (YI, YII and YIII) and all other invasive serogroup Y isolates, shown by (A) number of isolates and (B) incidence, Sweden, $2000-2010(n=85)$

A

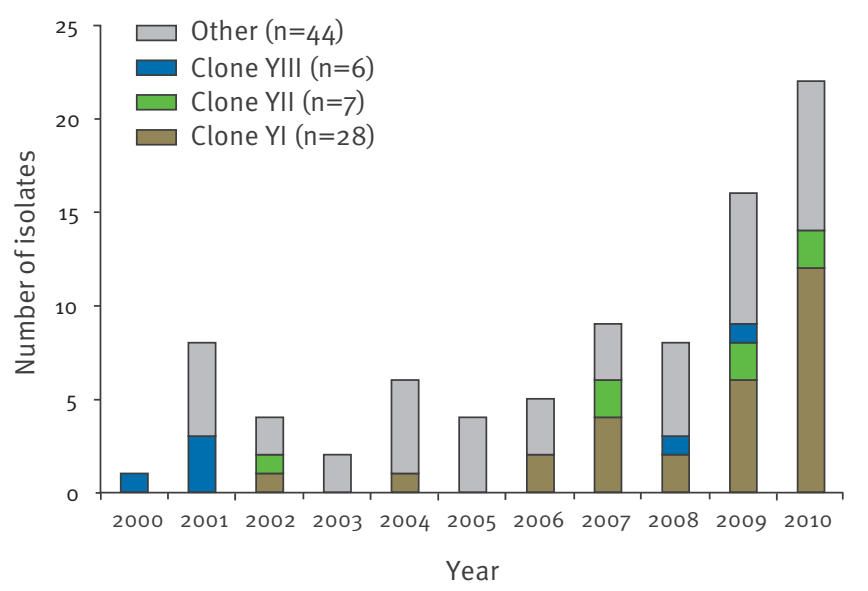

B

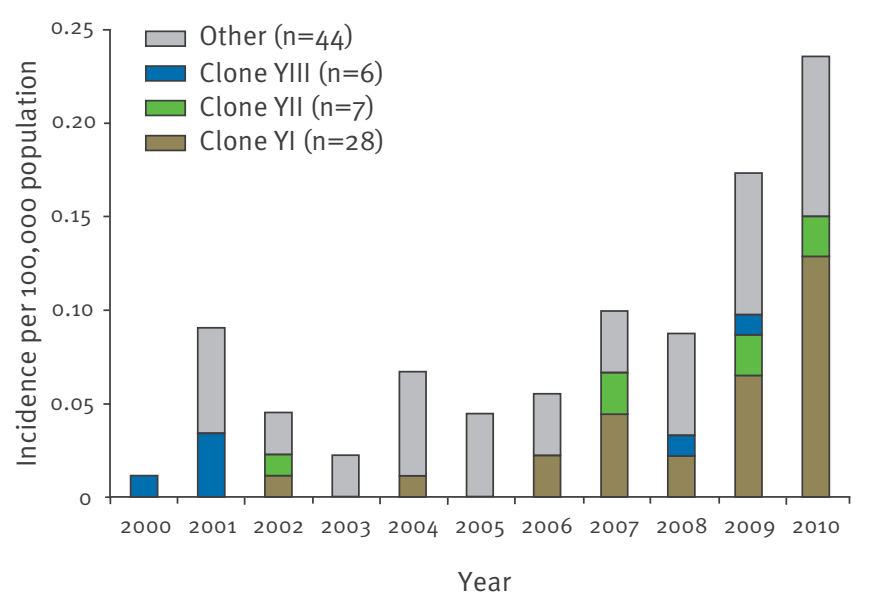


resistant, with genosubtype $\mathrm{P}_{1} \cdot 5-2,10-1,36-2$, ST 23 (cc23), porB allele 3-36, fet $A$ allele $\mathrm{F} 4-1, \mathrm{fHbp}$ allele 25 and penA allele 22. The second most frequent (Clone YII; $n=7)$ was sulfadiazine susceptible, with genosubtype $\mathrm{P} 1.5-1,2-2,36-2$, ST 23 (cc 23), porB allele 2-55, fet $A$ allele $\mathrm{F}_{5}-8$, $f H b p$ allele 25 and penA allele 22. The third most frequent (Clone YIII; $n=6$ ) was sulfadiazine susceptible, with genosubtype $\mathrm{P}_{1} \cdot 5-1,2-2,36-2, \mathrm{ST}_{2} 3$ (cc23), porB allele 3-36, fet $A$ allele $\mathrm{F}_{5}-8$, fHbp allele 25 and penA allele 1 . The remaining 44 isolates included clones of two to five identical isolates $(n=21)$ and 23 isolates with individual genetic profiles.

\section{Distribution of clones and other isolates}

Compilation of the epidemiological data on the isolates and genetic characterisation data generated a pattern of the distribution of the clones over time (Figure 2). There was no indication of an epidemiological link between the notified cases. The age distribution of patients with invasive disease caused by the three most common clones and all other serogroup $Y$ isolates is shown in Figure 3. An increase of invasive disease caused by Clone YI was seen in young adults (aged 20-29 years). Overall, this clone was significantly more prone ( $p<0.05$ Mann-Whitney U test) to cause disease in a younger age group ( $\neg$ median age: 47 years; interquartile range: $20-70$ ), compared with the isolates with other genetic profiles. These other clones and isolates had the usual pattern of serogroup $Y$ regarding age groups affected, i.e. they affected mainly elderly people (median age: 72 years; interquartile range: 36-84).

We also studied the geographical distribution of the three most common serogroup $Y$ clones and all other serogroup $Y$ isolates, which showed a higher incidence of Clone $\mathrm{YI}$ in young adults (aged 15-25 years) in the central parts of Sweden (data not shown). No such

\section{FIGURE 3}

Age distribution of patients with invasive meningococcal disease caused by the three most common Neisseria meningitidis serogroup Y clones (YI, YII and YIII) and all other invasive serogroup Y isolates, Sweden, 2000-2010 $(\mathrm{n}=85)$

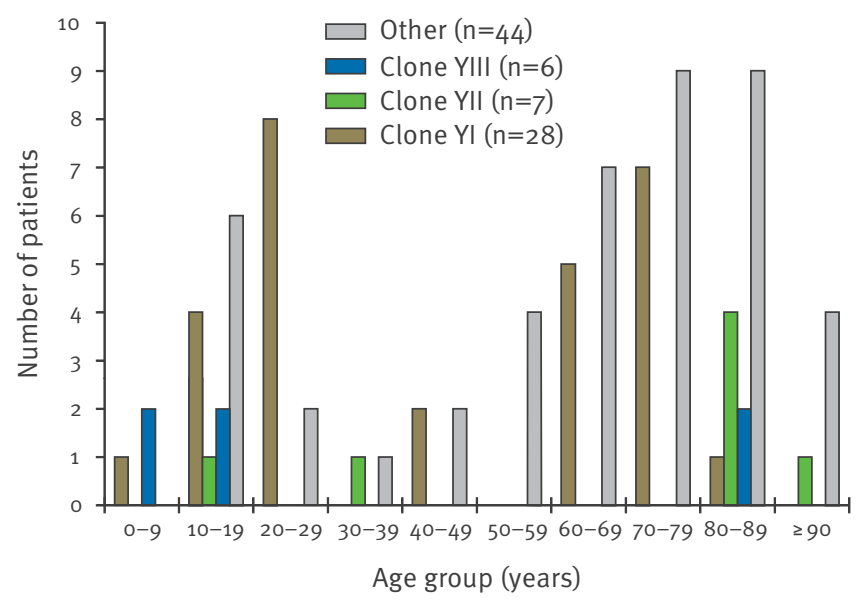

geographical pattern was seen for elderly cases with invasive isolates of Clone $\mathrm{YI}$ or cases with Clone YII, Clone YIII or any of the other isolates.

The mortality rate among patients with disease caused by serogroup $Y$ was $13 \%$ (11 of 85 ). Two of the 11 deceased patients were infected with Clone YI.

The genes that displayed the highest variability in the minimum-spanning trees (porA, por $B$ and fet $A$ ) are shown in Figure 4; the penA and $\mathrm{fHbp}$ genes were found to be fairly conserved (data not shown). These findings were further confirmed by the D-indices calculated for the genes used in MLST, and $\operatorname{por} A, \operatorname{por} B, f e t A$, fHbp and penA genes (Table 2).

\section{Discussion and conclusion}

Our results show that the most common invasive $N$. meningitidis serogroup $\mathrm{Y}$ isolate, clone $\mathrm{YI}$, was partly responsible for the increase of meningococcal disease caused by this serogroup in Sweden in recent years. This clone was prevalent at the beginning of the $2000 \mathrm{~s}$, with one isolate being identified in both 2002 and 2004 (incidence of 0.01 per 100,000 population in each year). The number of isolates gradually increased, from two isolates in 2006 to a peak of 12 in 2010 (incidence of 0.02 and 0.13 , respectively, per 1000,000 population). No outbreaks or clusters of cases due to serogroup $Y$ infection were noted in Sweden during 2000 to 2010.

The reasons for the increased incidence of invasive disease caused by Clone YI, compared with the other clones and isolates, among younger people are unclear. However, a similar pattern for disease due to serogroup $Y$ has been observed in Canada, where the proportion of cases with this serogroup in the age group 10-19 years increased from $11.8 \%$ in 1999 to $26.3 \%$ in 2003 , with a peak of $41.4 \%$ in 2001 [6]. In the US, however, no such increase has been observed among persons aged 15-24 years [25].

\section{TABLE 2}

Discrimination indices for sequence types and porA, por $B$, fet $A, f H b p$, penA genes, invasive Neisseria meningitidis serogroup Y isolates, Sweden, 2000-2010 $(n=85)$

\begin{tabular}{|l|c|c|c|}
\hline Target & $\begin{array}{c}\text { Number of } \\
\text { types }\end{array}$ & $\begin{array}{c}\text { Number (\%) of } \\
\text { isolates with the } \\
\text { most common } \\
\text { type }\end{array}$ & $\begin{array}{c}\text { Discrimination } \\
\text { index } \\
(95 \% \mathrm{Cl})\end{array}$ \\
\hline porA & 15 & $45(53)$ & $0.65(0.57-0.74)$ \\
\hline fetA & 7 & $53(62)$ & $0.55(0.45-0.65)$ \\
\hline porB & 9 & $58(68)$ & $0.51(0.39-0.62)$ \\
\hline $\begin{array}{l}\text { Sequence } \\
\text { types }\end{array}$ & 12 & $66(78)$ & $0.39(0.26-0.53)$ \\
\hline penA & 4 & $72(85)$ & $0.27(0.15-0.39)$ \\
\hline fHbp & 6 & $78(92)$ & $0.16(0.5-0.26)$ \\
\hline
\end{tabular}




\section{FIGURE 4}

Minimum-spanning trees based on multilocus sequence typing of all invasive Neisseria meningitidis serogroup Y isolates, Sweden, 2000-2010 ( $\mathrm{n}=85)$

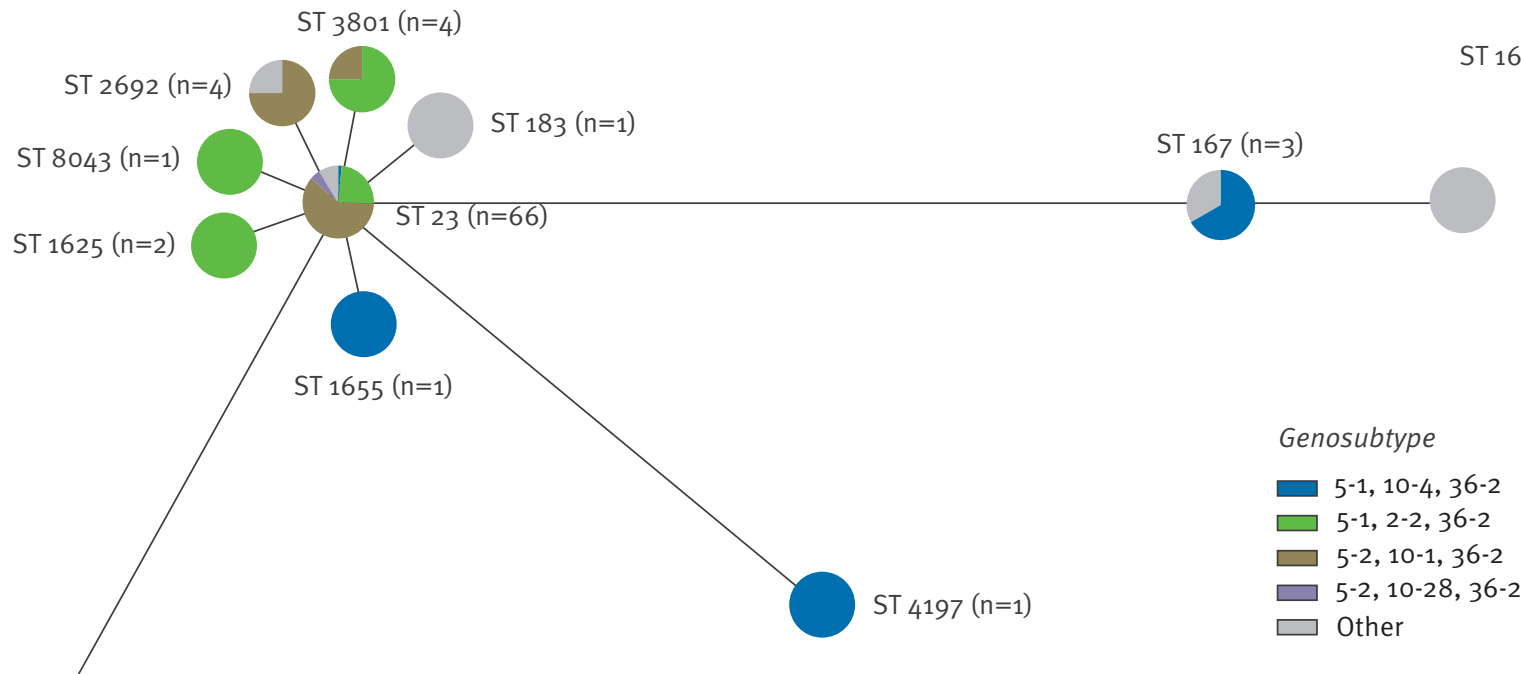

ST $1466(n=1)$
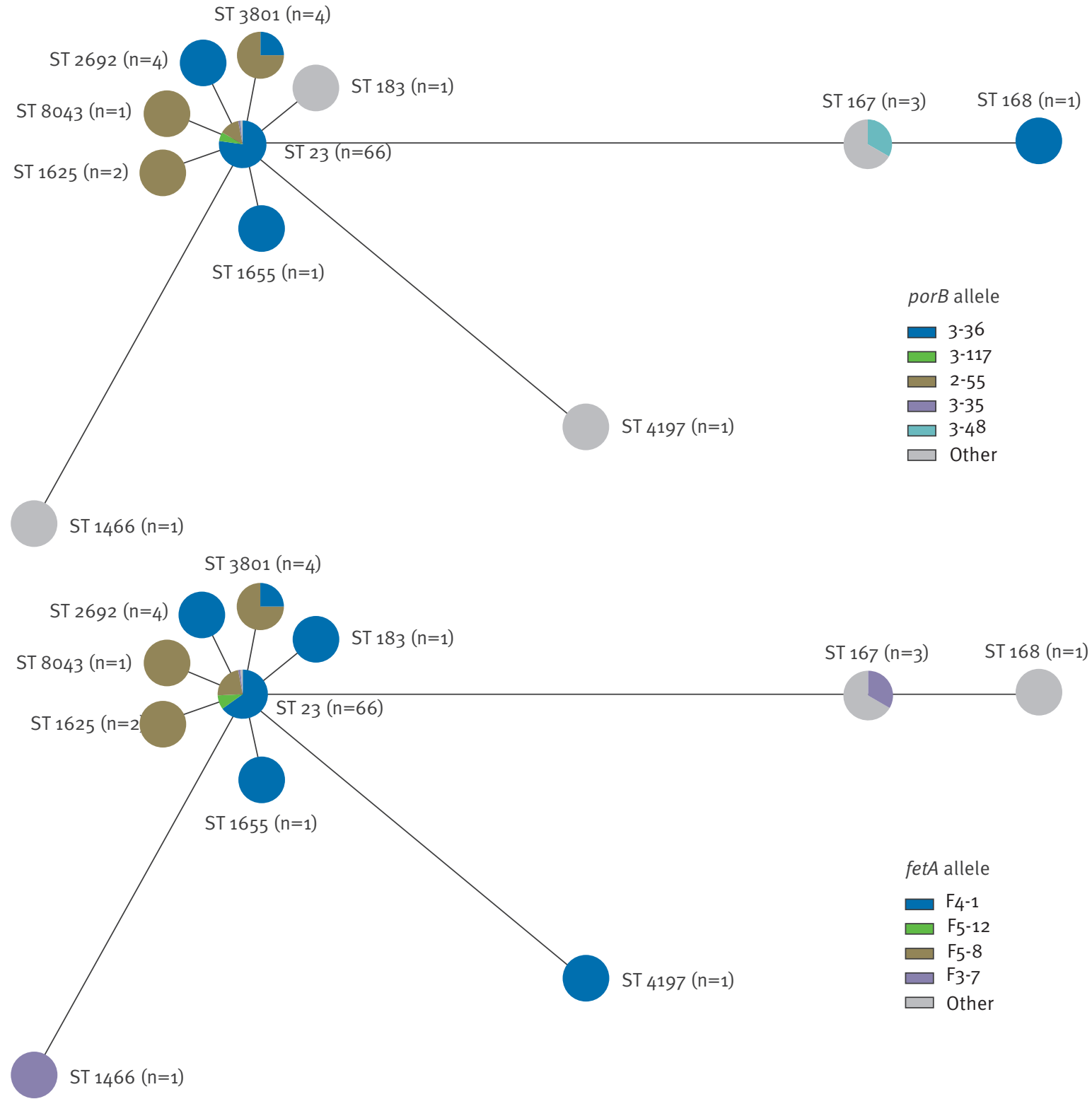

The circles denote different sequence types. The genosubtype (porA) and porB and fet $A$ alleles are distributed over the different sequence types. 
The second and third most common clones in Sweden, YII and YIII, consisted of few isolates per clone, which made the age distribution for cases with these clones difficult to interpret. It is interesting to note that in Maryland, US, two clones with different antigenic profiles seemed to be responsible for the increased number of invasive $N$. meningitidis serogroup $Y$ isolates in the 1990 s and that the clones are differently distributed over time [25]. The first, which shares parts of the genetic profile of clones YII and YIII in our study (cc23; P1.5-1,2-2; F5-8), was mainly responsible for early cases (before 1998), while the other clone, with the same genetic profile as the dominant Clone $\mathrm{YI}$ in our study (cc23; P1.5-2,10-1; F4-1), has dominated since 1998. In the Czech Republic, the most common clone (cc23; P1.5-2,10-1,36-2; F4-1) and second most common clone (cc23; P1.5-1,2-2,36-2; $\mathrm{F}_{5}-8$ ) present indistinguishable porA and fet $A$ profiles and ST as the most common clones in Sweden, in the same order of frequency [8].

Clone $\mathrm{YI}$ does not seem to be related to increased mortality, although a difference would be difficult to detect because of the low incidence of meningococcal disease in general. The mortality rate of all the studied invasive serogroup $Y$ isolates $(13 \%)$ is within the range of the overall global mortality rate for meningococcal disease caused by all serogroups (about 10\%) [2], but is considerably lower than the mortality rate for disease due to serogroup $Y$ in Sweden in 1995 to 2005 (17\%) [26]. The decreased mortality rate may be the result of the increased incidence of Clone $\mathrm{Yl}$ in a younger age group (median age: 47 years).

The emergence of serogroup $\mathrm{Y}$ Clone $\mathrm{YI}$ is presumably due to multiple underlying factors, but the most probable is that it is an epidemiologically competent clone with increased pathogenicity. It is less likely that host adaptive immunity has decreased in different geographical areas concurrently. It is also possible that Clone $\mathrm{YI}$ possesses characteristics that lead to increased transmission efficiency. This possibility is difficult to investigate and no dynamic carriage studies have been performed in Sweden. However, in some carriage studies from elsewhere in Europe and the US, it was found that serogroup $Y$ was one of the most common serogroups among carrier isolates [27-30].

The results from the minimum-spanning tree and $\mathrm{D}$-indices indicate that in the case of serogroup $\mathrm{Y}$, targeting the penA and $\mathrm{fH} b p$ genes yields a less discriminatory pattern than MLST or targeting the porA, por $B$ and fetA genes. Although penA and $\mathrm{FHbp}$ seem to be too conserved to be of use for further differentiation of clones, these genes are still of interest for following penicillin resistance and the effect of promising novel vaccines that include the factor $\mathrm{H}$-binding protein. Although none of the individual genes in our study reached the recommended discrimination level of $90 \%$ [21], a combination of target genes and inclusion of other serogroups probably could. However, using only typing tools with high $\mathrm{D}$-indices may result in missing isolates belonging to the same clone. Similarly, the apparent frequency of the clone would increase if only stable genes are targeted, as in MLST. Therefore, Clone $\mathrm{YI}$ is defined by what we think is an informative and appropriate number of target genes, which is also somewhat supported by the minimum-spanning trees and D-indices.

Further research could be carried out using characterisation tools that would increase the discrimination, such as variable number tandem repeat (VNTR) analysis, to provide more information about the clone. However, it will also be a challenge to customise the characterisation tools, to achieve an appropriate balance between the information required for the particular investigation and the time and cost.

In conclusion, the clone responsible for the recent emergence of $N$. meningitidis serogroup $\mathrm{Y}$ isolates was identified and described in this study. To achieve a complete epidemiological profile of the clone, it would be useful to examine invasive serogroup $Y$ isolates from before 2000 and carry out additional typing. The general increase of meningococcal disease due to $N$. meningitidis serogroup $Y$ has an important public health implication because this is one of the serogroups that is covered by available vaccines for meningococcal disease.

\section{References}

1. Stephens DS, Greenwood B, Brandtzaeg P. Epidemic meningitis, meningococcaemia, and Neisseria meningitidis. Lancet. 2007;369(9580):2196-210.

2. Caugant DA. Genetics and evolution of Neisseria meningitidis: importance for the epidemiology of meningococcal disease. Infect Genet Evol. 2008;8(5):558-65.

3. Harrison LH, Trotter CL, Ramsay ME. Global epidemiology of meningococcal disease. Vaccine. 2009;27 Suppl 2:B51-63.

4. Rosenstein NE, Perkins BA, Stephens DS, Lefkowitz L, Cartter ML, Danila R, et al. The changing epidemiology of meningococcal disease in the United States, 1992-1996. J Infect Dis. 1999;180(6):1894-901.

5. Centers for Disease Control and Prevention (CDC). Active bacterial core Surveillance $(A B C s)$ report. Emerging Infections Program Network. Neisseria meningitidis, 2009. Oct2010 File 17 Nov 2010. Available from: http://www.cdc.gov/abcs/reportsfindings/survreports/mening09.pdf

6. Tsang RS, Henderson AM, Cameron ML, Tyler SD, Tyson S, Law DK, et al. Genetic and antigenic analysis of invasive serogroup Y Neisseria meningitidis isolates collected from 1999 to 2003 in Canada. J Clin Microbiol. 2007;45(6):1753-8.

7. Inés Agudelo C, Sanabria OM, Ovalle MV. Serogroup $Y$ meningococcal disease, Colombia. Emerg Infect Dis. 2008;14(6):990-1.

8. The European Meningococcal Disease Society (EMGM). 11th EMGM meeting, 18-20 May 2011, Ljubljana Slovenia. Poster abstracts Po35, 037-040.

9. Jolley KA, Brehony C, Maiden MC. Molecular typing of meningococci: recommendations for target choice and nomenclature. FEMS Microbiol Rev. 2007;31(1):89-96.

10. European Centre for Disease Prevention and Control (ECDC). Case definition. 2008 EU Case Definition (2008/426/EC) for meningococcal disease, invasive. Stockholm: ECDC. Available from: http://www.ecdc.europa.eu/EN/ACTIVITIES/ SURVEILLANCE/EU_IBD/Pages/Case_definition.aspx

11. Olcén $P$, Danielsson D, Kjellander J. The use of protein A-containing staphylococci sensitized with anti-meningococcal antibodies for grouping Neisseria meningitidis and demonstration of meningococcal antigen in cerebrospinal fluid. Acta Pathol Microbiol Scand B. 1975;83(4):387-96. 
12. Mölling P, Jacobsson S, Backman A, Olcén P. Direct and rapid identification and genogrouping of meningococci and porA amplification by LightCycler PCR. J Clin Microbiol. 2002;40(12):4531-5.

13. Tettelin H, Saunders NJ, Heidelberg J, Jeffries AC, Nelson $\mathrm{KE}$, Eisen JA, et al. Complete genome sequence of Neisseria meningitidis serogroup B strain $\mathrm{MC}_{5} 8$. Science. 2000;287(5459):1809-15.

14. Primers used for MLST of Neisseria. Neisseria Multi Locus Sequence Typing website (http://pubmlst.org/ neisseria/) developed by Keith Jolley and sited at the University of Oxford. [Accessed 4 Mar 2011]. Available from: http://pubmlst.org/ neisseria/info/primers.shtml

15. Taha MK, Vazquez JA, Hong E, Bennett DE, Bertrand S, Bukovski S, et al. Target gene sequencing to characterize the penicillin $\mathrm{G}$ susceptibility of Neisseria meningitidis. Antimicrob Agents Chemother. 2007;51(8):2784-92.

16. Thulin $\mathrm{S}$, Olcen $\mathrm{P}$, Fredlund $\mathrm{H}$, Unemo $\mathrm{M}$. Total variation in the penA gene of Neisseria meningitidis: correlation between susceptibility to beta-lactam antibiotics and penA gene heterogeneity. Antimicrob Agents Chemother. 2006;50(10):3317-24.

17. Urwin R. Nucelotide sequencing of antigen genes of Neisseria meningitidis. In: Pollard AJ, Maiden MCJ, editors. Meningococcal disease. New Jersey: Humana Press; 2001. p. 165 .

18. Jacobsson S, Thulin S, Mölling P, Unemo M, Comanducci M, Rappuoli $R$, et al. Sequence constancies and variations in genes encoding three new meningococcal vaccine candidate antigens. Vaccine. 2006;24(12):2161-8.

19. Brehony C, Wilson DJ, Maiden MC. Variation of the factor $\mathrm{H}$-binding protein of Neisseria meningitidis. Microbiology. 2009;155(Pt 12):4155-69.

20. Sequence query. Neisseria Multi Locus Sequence Typing website (http://pubmlst.org/ neisseria/) developed by Keith Jolley and sited at the University of Oxford. [Accessed 4 Mar 2011]. Available from: http://pubmlst.org/perl/bigsdb/bigsdb. pl?db=pubmlst_neisseria_seqdef\&page=sequenceQuery

21. Hunter PR, Gaston MA. Numerical index of the discriminatory ability of typing systems: an application of Simpson's index of diversity. J Clin Microbiol. 1988;26(11):2465-6.

22. Grundmann H, Hori S, Tanner G. Determining confidence intervals when measuring genetic diversity and the discriminatory abilities of typing methods for microorganisms. J Clin Microbiol. 2001;39(11):4190-2.

23. MLST data analysis. Neisseria Multi Locus Sequence Typing website (http://pubmlst.org/ neisseria/) developed by Keith Jolley and sited at the University of Oxford. [Accessed 9 Mar 2011]. Available from: http://pubmlst.org/analysis/

24. McEllistrem MC, Kolano JA, Pass MA, Caugant DA, Mendelsohn $A B$, Fonseca Pacheco $A G$, et al. Correlating epidemiologic trends with the genotypes causing meningococcal disease, Maryland. Emerg Infect Dis. 2004;10(3):451-6.

25. Harrison LH, Jolley KA, Shutt KA, Marsh JW, O'Leary M, Sanza LT, et al. Antigenic shift and increased incidence of meningococcal disease. J Infect Dis. 2006;193(9):1266-74.

26. Jacobsson S, Olcén P, Löfdahl M, Fredlund H, Mölling P. Characteristics of Neisseria meningitidis isolates causing fatal disease. Scand J Infect Dis. 2008;40(9):734-44.

27. Bidmos FA, Neal KR, Oldfield NJ, Turner DP, Ala'Aldeen DA, Bayliss CD. Persistence, replacement, and rapid clonal expansion of meningococcal carriage isolates in a 2008 university student cohort. J Clin Microbiol. 2011;49(2):506-12.

28. Yazdankhah SP, Kriz P, Tzanakaki G, Kremastinou J, Kalmusova J, Musilek M, et al. Distribution of serogroups and genotypes among disease-associated and carried isolates of Neisseria meningitidis from the Czech Republic, Greece, and Norway. J Clin Microbiol. 2004;42(11):5146-53.

29. Glitza IC, Ehrhard I, Müller-Pebody B, Reintjes R, Breuer $\mathrm{T}, \mathrm{Ammon} \mathrm{A}$, et al. Longitudinal study of meningococcal carrier rates in teenagers. Int J Hyg Environ Health. 2008;211(3-4):263-72.

30. Claus H, Maiden MC, Wilson DJ, McCarthy ND, Jolley KA, Urwin $\mathrm{R}$, et al. Genetic analysis of meningococci carried by children and young adults. J Infect Dis. 2005;191(8):1263-71. 